\title{
Gut feelings a strong influence on physician decisions
}

Cite as: CMAJ 2018 August 20;190:E998-9. doi: 10.1503/cmaj.109-5647

Posted on cmajnews.com on August 2, 2018.

A rtificial intelligence and robotics may outperform doctors in some areas, but a new study highlights the important role human intuition plays in medical decisions.

Computer scientists at the Massachusetts Institute of Technology (MIT) analyzed 10 years of data on patients in intensive care and found that doctors' gut feelings about how their patients were doing influenced how many tests they ordered. The researchers collected information on all factors a doctor might consider in deciding to order tests, including a patient's age, gender, disease type and severity. They also measured how doctors felt about their patients by analyzing care notes using an algorithm that scores text for positive and negative sentiment.

When doctors were more pessimistic about a patient's condition, they tended to order more tests, up to a point. If they felt very negative about the patient's prognosis, they ordered fewer tests. This effect was strongest at the beginning of a patient's hospital stay, when doctors had less medical information to go on, and declined over time.

According to study lead Mohammad Ghassemi, "there's something about a doctor's experience, and their years of training and practice, that allows them to know in a more comprehensive sense, beyond just the list of symptoms, whether you're doing well or not."

The study helps to explain why there can be so much variation in the use of medical resources, even in similar cases. Whether the doctors' hunches about their patients were correct is another matter. Modern medicine has tended to value more conscious, deliberate analysis over intuitive reasoning. Some doctors argue this has resulted in overly simplistic "tick box" care. Clinical guidelines may define the most likely presentation, treatment and outcome of a disease but may not apply in unique cases.

Research shows that the earliest impressions a person forms when confronted with a problem can often be more accurate than later analysis. Studies of physicians demonstrate that the best pre- dictor of diagnostic accuracy is having a hunch about a patient's condition in the first minutes of an encounter. One study published in BMJ found that a doctor's gut feeling that something was wrong when treating a child in primary care can have greater diagnostic value than most signs and symptoms.

Although it's often assumed these hunches draw from clinical experience, it's not clear how much experience

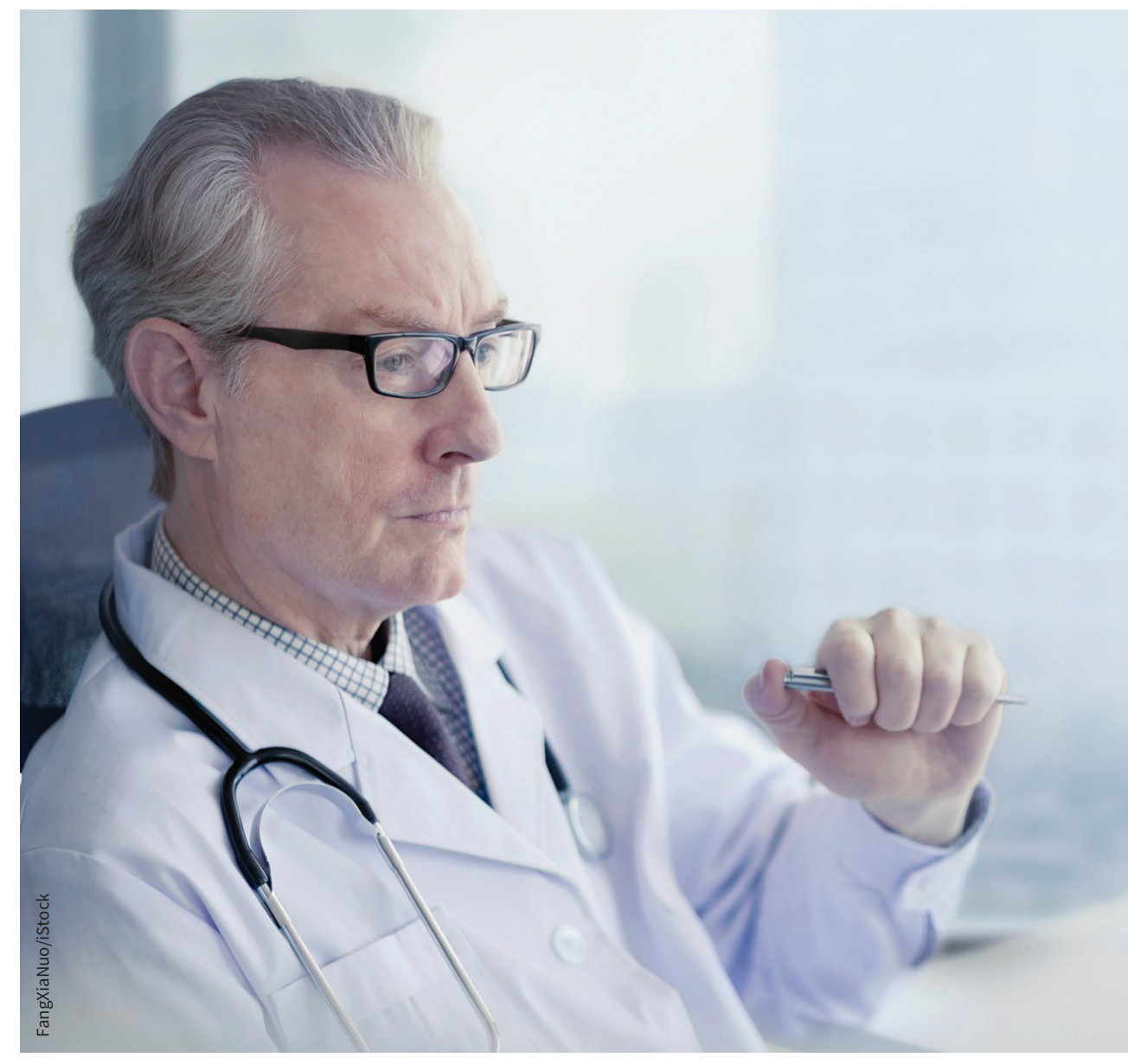

Doctors rely on intuition, not just data, when deciding what tests to order. 
matters. In the BMJ study, a doctor's intuition about a child's condition was strongly influenced by how much the parents were concerned, but the clinician's level of experience made no difference to the diagnostic value of the hunch.

Other studies have shown that instructing medical trainees to use intuition can lead to equal or greater accuracy in diagnosis. This may be because clinical intuition has more to do with empathy than expertise. One recent study found that general practitioners who scored highest on empathy were four times as likely to report using gut feelings in practice as those who scored lowest on empathy.
It remains to be seen whether this intuition is a uniquely human dimension of care. According to MIT study coauthor Tuka Alhanai, "The question is, can you get the machine to do something like that?"

Lauren Vogel, CMAJ 